\title{
Estudo Prospectivo sobre a Cisteamina no Tratamento do Melasma
}

\author{
Prospective Study of Cysteamine in the Treatment of Melasma
}

\author{
Marília Dione Salvador Shibayama \\ Geraldo Neves de Albuquerque Maranhão ${ }^{1}$ \\ Werbeston Douglas de Oliveira ${ }^{1}$ \\ ${ }^{1}$ Universidade Federal do Amapá, Macapá, AP, Brasil
}

\begin{abstract}
Resumo
O tratamento de melasma, desordem pigmentar caracterizada por manchas escuras na pele, é difícil e demorado, pois não há cura, apenas controle. Conforme os últimos estudos na área de dermatologia, a Cisteamina, é um ativo que age como corretor de pigmentos, reduzindo a melanina da pele, corrigindo o melasma e a hiperpigmentação da pele. A Cisteamina é um despigmentante, ou seja, é um clareador com efeito antioxidante. Assim, este estudo teve como objetivo realizar um levantamento de informações sobre patentes relacionadas ao uso da Cisteamina como um despigmentador da pele com melasma. Utilizou-se o Mapeamento patentário, o Levantamento bibliográfico e a Discussão da Literatura. Percebeu-se que há baixa produção de artigos publicados no Brasil, sendo autores estrangeiros os que mais publicam sobre o tema pesquisado. Assim como nas bases de dados internacionais, o resultado da busca trouxe as diversas formas de se utilizar a Cisteamina, não somente na dermatologia, mas para tratamento capilar, infecções causadas por fungos e vírus, diabetes, cistinose, entre outros. Porém, especificamente para tratar melasma, ainda não há registro no Brasil.
\end{abstract}

Palavras-chave: Cisteamina. Melasma. Prospecção Tecnológica.

\begin{abstract}
The treatment of melasma, a pigmentary disorder characterized by dark patches on the skin, is difficult and timeconsuming because there is no cure, only control. According to the latest studies in the field of dermatology, Cysteamine is an active agent that acts as a pigment broker, reducing the melanin of the skin, correcting melasma and hyperpigmentation of the skin. Cysteamine is a depigmentant, a bleaching agent with an antioxidant effect. Thus, this study aimed to conduct a survey of information on patents related to the use of Cysteamine as a skin depigmenter with melasma. It was used the patent Mapping, Bibliographic Survey and Discussion of literature . It was noticed that there is low production of articles published in Brazil, with foreign authors being the ones who publish the most on the subject researched. As in the international databases, the search result brought the different ways of using Cysteamine, not only in dermatology, but for capillary treatment, infections caused by fungi and viruses, diabetes, cystinosis, among others. However, specifically to treat melasma, there is still no record in Brazil.
\end{abstract}

Keywords: Cysteamine. Melasma. Technological Prospection.

Área Tecnológica: Prospecções Tecnológicas de Assuntos Específicos. 


\section{Introdução}

Melasma é o surgimento de manchas escuras na pele, que normalmente aparecem no rosto e são produzidas pelo próprio organismo, decorrente da deposição aumentada de melanina, proteína que garante a coloração da pele e evita os danos da radiação ultravioleta no DNA. O transtorno resulta na formação de manchas castanho-escuras ou marrom-acinzentadas, com limites bem demarcados, mas formato irregular (VARELLA, 2018).

Melasma é uma doença adquirida pelo excesso de pigmentação da pele que mais comumente se apresenta como máculas de cores marrom a cinza distribuídas simetricamente e manchas reticuladas que têm uma predileção pela face. Raramente pode aparecer no pescoço, parte superior do tórax, parte superior das costas e antebraços extensores. Com base na profundidade da deposição de melanina, o melasma pode ser classificado em quatro subtipos - epidérmico, dérmico, misto ou indeterminado (HUERTH; SHAHZEB; CALLENDER, 2019).

É um distúrbio de pigmentação adquirido que consiste no aparecimento de máculas hiperpigmentadas irregularidades que afetam áreas expostas ao sol, especialmente o rosto, onde o paciente pode adotar um padrão no centrofacial, malar ou mandibular. Também pode aparecer em outras áreas como os antebraços e o decote. Isso afeta especialmente as mulheres $e$ todos os grupos étnicos, mas é mais prevalente nos fototipos escuros (MUNOZ; ESTEBANEZ; ESTRADA, 2010).

Não há uma causa definida, mas muitas vezes esta condição está relacionada ao uso de anticoncepcionais femininos, à gravidez e, principalmente, à exposição solar. Conforme os últimos estudos na área de dermatologia, a Cisteamina, é um ativo que age como corretor de pigmentos, reduzindo a melanina da pele, corrigindo o melasma e a hiperpigmentação da pele. A Cisteamina é um despigmentante, ou seja, é um clareador com efeito antioxidante. A nova substância beneficia, principalmente, os pacientes que apresentam resistência à fórmula clássica de tratar a doença, uma combinação de hidroquinona, ácido retinoico e corticoide (HSU; MAHDI; POURAHMADI, 2013).

O tratamento de melasma, desordem pigmentar caracterizada por manchas escuras na pele, é um dos grandes desafios da Dermatologia, pois até hoje não há cura e sim, apenas controle. As áreas afetadas (geralmente no rosto) pelo melasma acumulam melanina, uma proteína responsável por pigmentar pele, olhos e cabelos. Os produtores de melanina passam a agir de forma desordenada em quem tem melasma, produzindo pigmentos em áreas delimitadas, deixando a pele com um tom desigual, causando impacto negativo sobre a autoestima e a qualidade de vida dos portadores do transtorno, principalmente em pessoas que moram em regiões em que a incidência solar é bastante alta, já que os raios ultravioletas, presentes na luz solar, aumentam a produção de melanina, agravando a situação (GHEISARI; DADKHAHFAR; OLAMAEI, 2019).

O presente estudo tem como objetivo realizar uma análise prospectiva das patentes de Cisteamina depositadas no Brasil e no exterior, levando em consideração os principais depositantes, em uma evolução temporal de 20 anos, e nele pretende-se apresentar um mapeamento tecnológico dos pedidos de patente relacionados à Cisteamina, com a finalidade de identificar quantitativamente a evolução do número de pedidos, os países que se destacam como depositantes no exterior, além dos códigos de International Patent Classification - IPC predominantes, que por sua vez remetem ao tipo de produto/processo objeto do pedido de proteção, no caso, a Cisteamina. 


\section{Metodologia}

Utilizou-se para a realização da prospecção, o mapeamento patentário com o uso do sistema de base de dados do Orbit Intelligence for Patent, o qual possui cobertura de publicações de quase uma centena de países e autoridades de patentes. Fez-se o uso do termo Cisteamina nos campos de busca de "título", "resumo", "reinvidicações" e "assunto". Optou-se por realizar a busca pelo nome em inglês da Cisteamina, no caso cysteamine. Esses dados foram coletados durante o mês de julho de 2019. Outra pesquisa foi realizada na base de dados do Instituto Nacional da Propriedade Industrial - INPI. Além disso, para complementação dos dados, foram utilizadas informações obtidas em artigos acadêmicos publicados em periódicos disponíveis na plataforma CAPES e no Repositório Latino-Americano da Universidade do Chile, no intuito de realizar o levantamento bibliográfico.

Aplicou-se o Mapeamento patentário, Levantamento bibliográfico e Discussão da Literatura. Fez-se o uso do termo "cysteamine" AND "melasma" nos campos de busca de "título", "resumo", "reinvidicações" e "assunto". Através da classificação IPC, pesquisou-se as seguintes classificações: A61k-103/10, A61k-38/31 e A61k-51/06 que compreendem áreas da Ciência médica, como dispositivos ou métodos especialmente adaptados para dar aos produtos farmacêuticos formas físicas determinadas. Essas classificações foram utilizadas a partir do resultado da busca das patentes internacionais, pois as empresas que possuem registro de patente de cisteamina como tratamento do melasma usam essas referidas classificações.

\section{Resultados e Discussão}

\subsection{Mapeamento Patentário}

Para o mapeamento patentário, manuseando a base de dados Orbit Intelligence for Patent percebe-se, no Gráfico 1, um resultado de 1.639 de invenções patenteadas com a substância cysteamine, sendo $15 \%$ de propriedade dos melhores depositantes. Em relação ao status da patente, observa-se o seguinte: $25,38 \%$ já foram caducadas, $31,73 \%$ concedidas, $20,81 \%$ pendentes, $7,75 \%$ revogadas e $14,34 \%$ expiradas.

Gráfico 1 - Status da patente

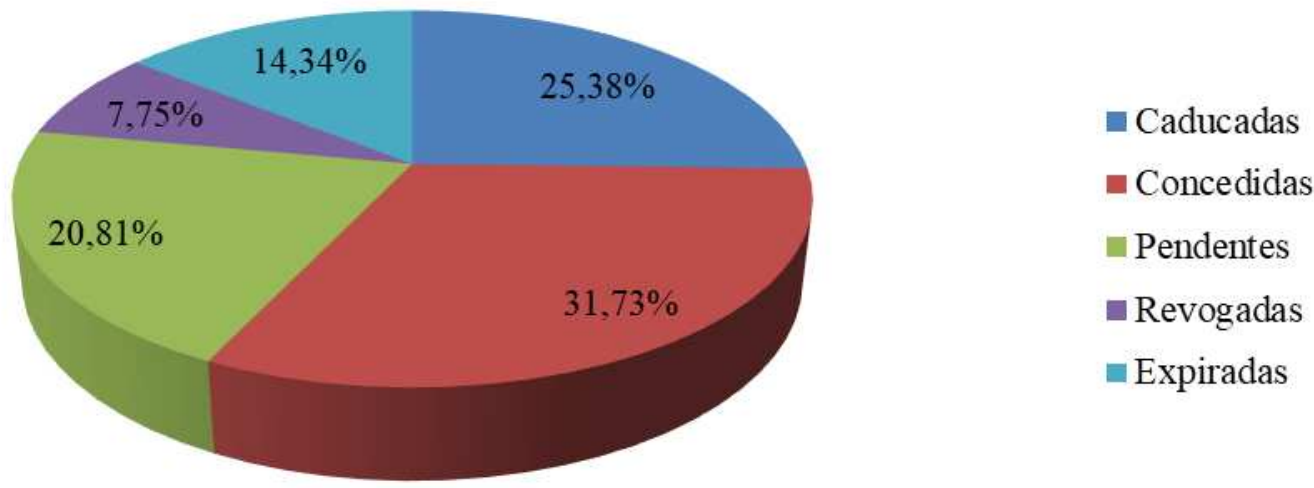

Fonte: Elaborado pelos autores deste artigo, a partir de Orbit Intelligence (2019) 
Observando-se as patentes de famílias por destinatários, no Gráfico 2, a L'Oréal é a empresa que mais deposita registro de patente em diferentes países com o propósito de proteger uma mesma invenção. A principal característica da família de patentes é que o direito de prioridade do primeiro depósito é estendido aos demais nos diferentes países em que foram depositados.

Gráfico 2 - Patentes de famílias por destinatários

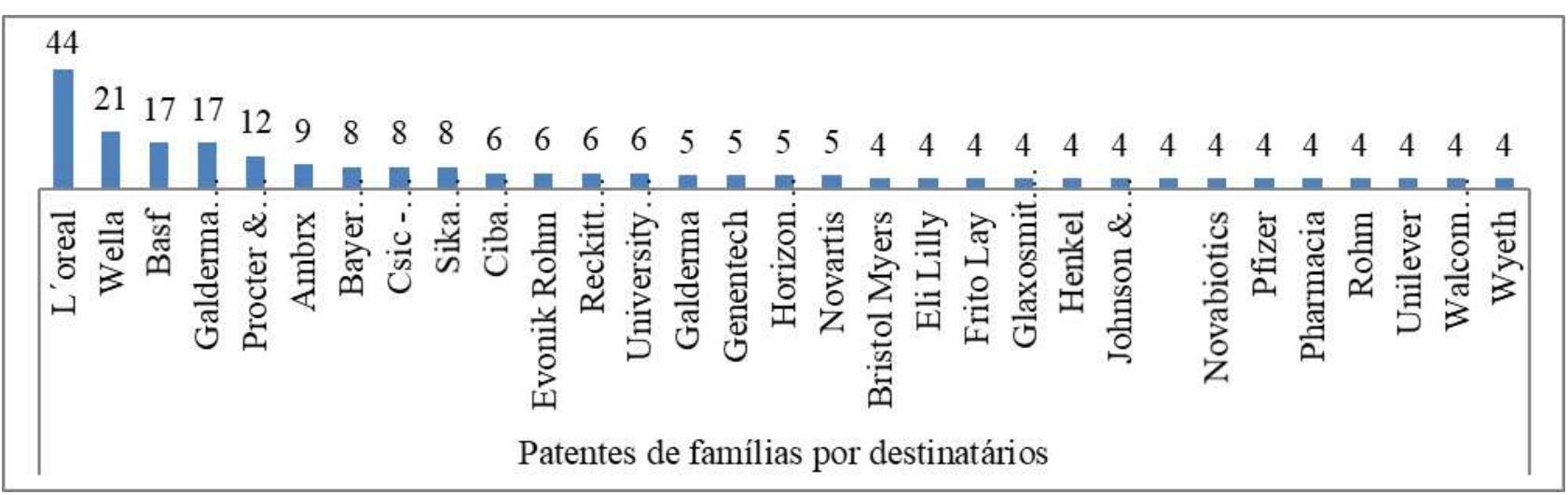

Fonte: Elaborado pelos autores deste artigo, a partir de Orbit Intelligence (2019)

Conforme o Gráfico 3, 2016 foi o ano que mais teve registro de patentes com a substância tópica Cisteamina. Já em 2019 ainda é baixo o registro, porém o sistema de patentes, não serve apenas como uma simples ferramenta de proteção dos ativos intangíveis, mas também como um instrumento analítico para as atividades de Inteligência Tecnológica, uma vez que as patentes podem ser consideradas as primeiras provas concretas de um novo produto ou processo industrial. Em outras palavras, a avaliação da atividade de patenteamento permite elaborar diversos indicadores os quais tornam possível, com um certo grau de confiança, identificar em quais países e empresas o desenvolvimento de determinadas tecnologias está estável ou progredindo.

Gráfico 3 - Evolução por ano do primeiro ano de aplicação

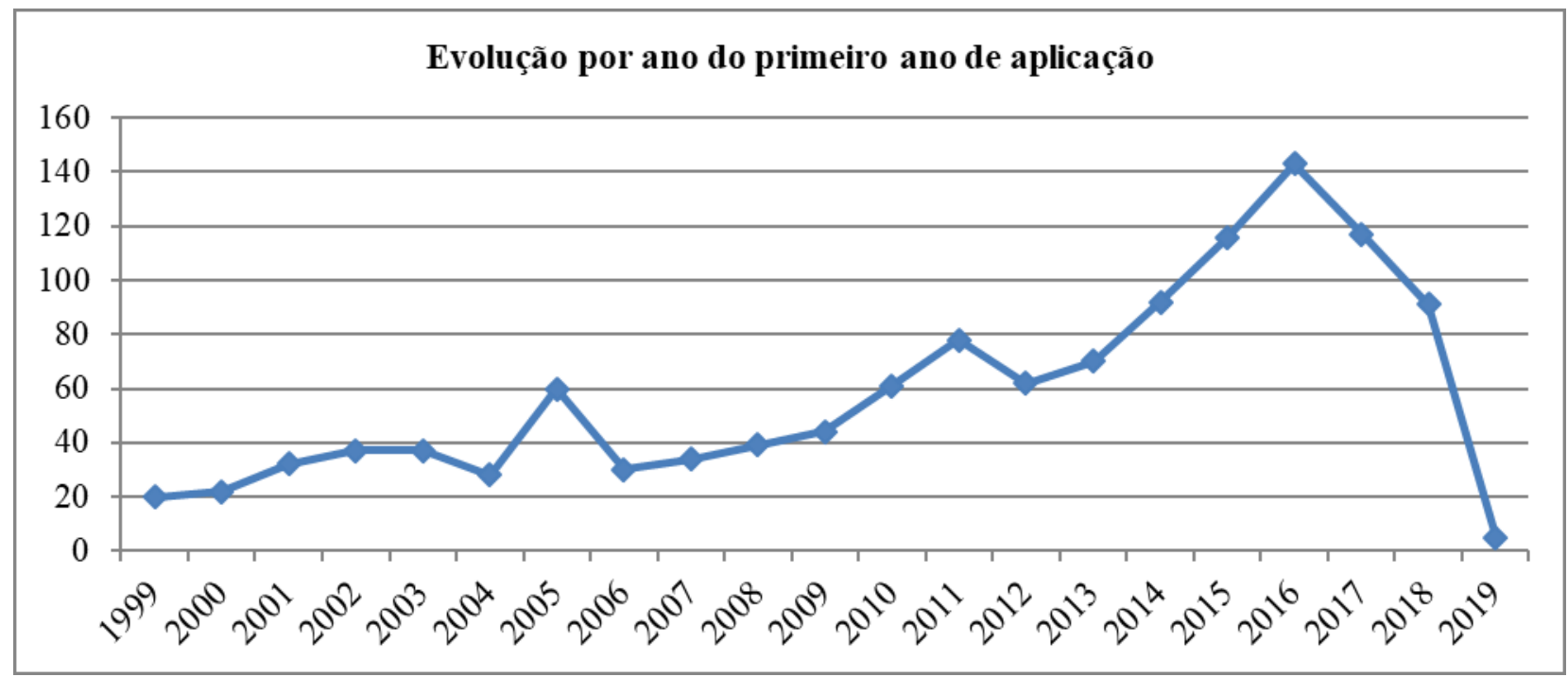

Fonte: Elaborado pelos autores deste artigo, a partir de Orbit Intelligence (2019) 
No Gráfico 4 foi feita uma análise dos dados conforme a classificação internacional de patentes, usando as classes A61k-103/10, A61k-38/31 e A61k-51/06 do IPC; e é possível notar que nos últimos 20 anos a classe A61k-103/10 (finalidade médica, odontológica e higiênico) é mais mencionada do que as outras duas pesquisadas, no caso A61k-38/31 (preparações medicinais de medicamentos) e A61k-51/06 (preparações contendo compostos macrooleculares). No resultado da busca a classe A61k 103/10 obteve um total de 713 resultados no período de 1999 até o ano atual, enquanto que a classe A61k 38/31 teve um total de 534 resultados e a classe A61k 51/06 resultou em 590 registros ao longo dos últimos 20 anos.

Gráfico 4 - Classificação Internacional de Patentes

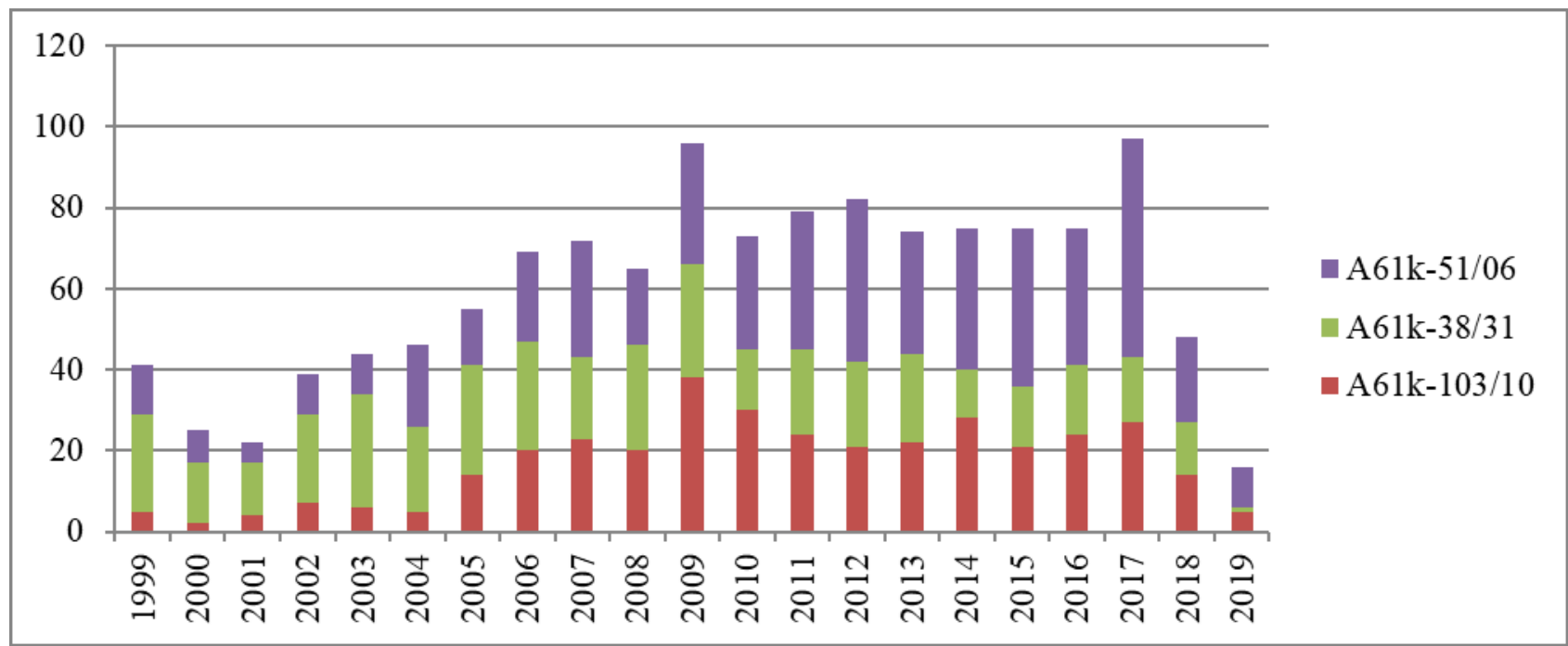

Fonte: Elaborado pelos autores deste artigo, a partir de Orbit Intelligence (2019)

De acordo com o Gráfico 5, China, Estados Unidos, Organização Europeia de Patentes, Japão e Coreia do Sul são os países que lideram a maior quantidade de registro de patentes, já que várias empresas de renome na área de cosméticos depositaram nesses lugares.

Gráfico 5 - Famílias de patentes por país de proteção

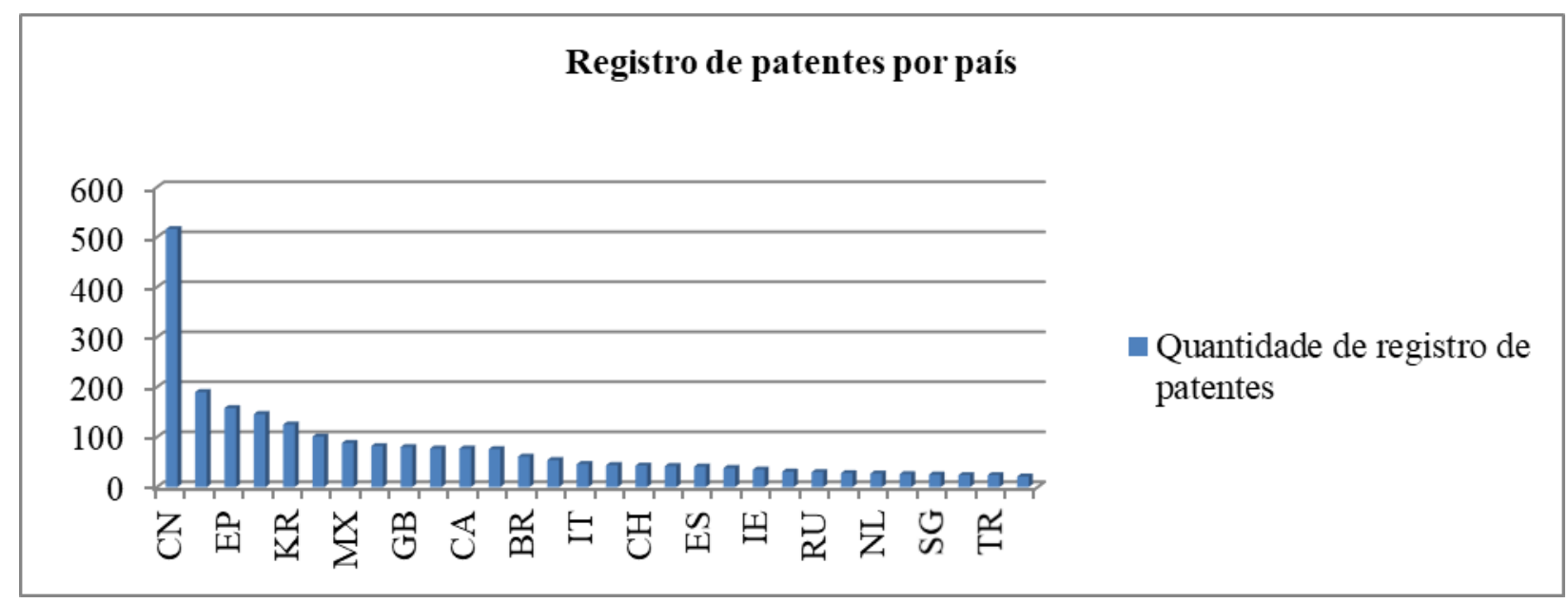

Fonte: Elaborado pelos autores deste artigo, a partir de Orbit Intelligence (2019) 
A proteção da propriedade intelectual, garantida pelas patentes, é altamente recomendada, pois pode gerar mais lucros de suas licenças, encorajar parcerias e, sem sombras de dúvidas, apresentar expressivas vantagens no mercado, já que impede outras empresas de utilizarem a sua invenção.

No Gráfico 6, o resultado de buscas conjuntas nas patentes internacionais com os conteúdos "Melasma" e "Cysteamine" teve como consequência 152 registros utilizando a cisteamina no ramo da dermatologia, sendo a empresa Galderma Research \& Development com mais registros.

Gráfico 6 - Patentes internacionais com o conteúdo "Melasma" e "Cysteamine"

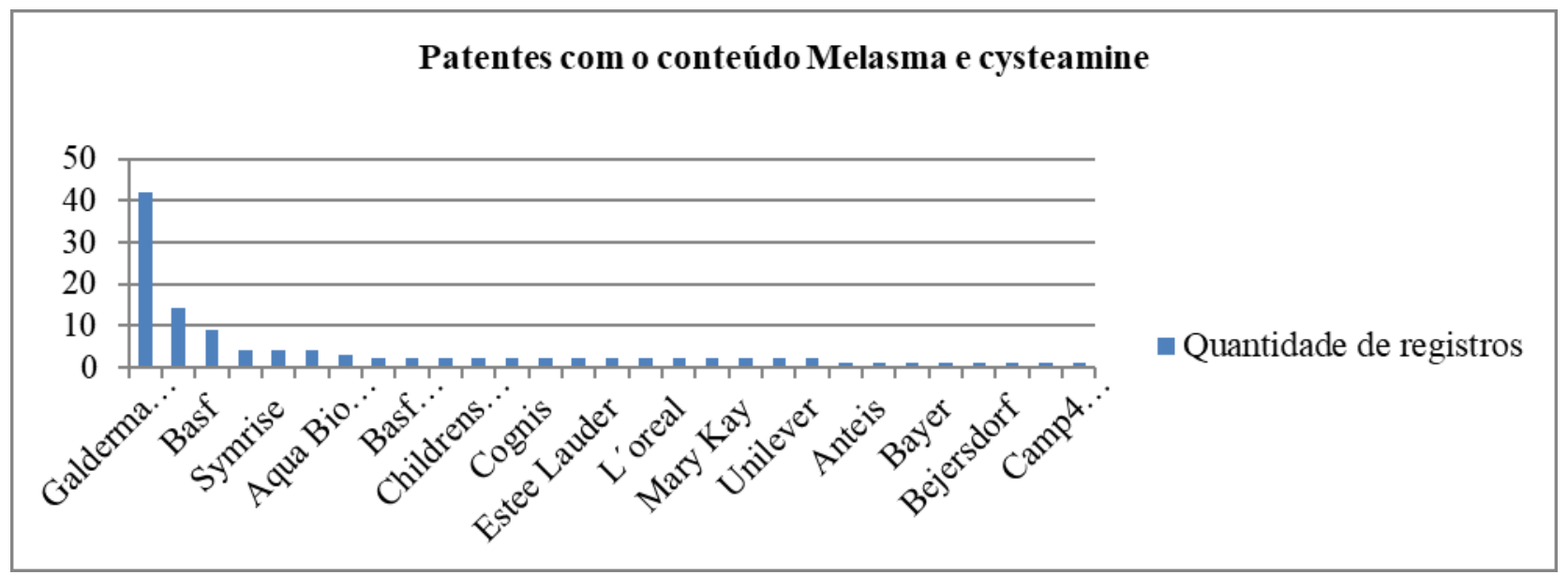

Fonte: Elaborado pelos autores deste artigo, a partir de Orbit Intelligence (2019)

No cenário nacional, na Figura 1, fez-se a busca com o termo Cisteamina na base de dados do INPI. Na pesquisa optou-se por verificar o termo Cisteamina no campo do resumo, título, nome do depositante e nome do inventor.

Figura 1 - Resultado da busca sobre a Cisteamina

[ Início | Ajuda? ]

» Consultar por: Base Patentes | Finalizar Sessão

\begin{tabular}{|c|c|c|}
\hline Pedido & Depósito & IPC \\
\hline BR 1120190060499 & 10/10/2017 FORMULAÇÕES DE CISTEAMINA E DERIVADOS DE CISTEAMINA & A61K 9/16 \\
\hline BR 1020170151263 & 14/07/2017 COMPOSIÇÕES COSMÉTICAS PARA MODELAGEM CAPILAR & A61K $8 / 46$ \\
\hline BR 1120180752215 & 06/06/2017 MICROPARTÍCULAS COMPREENDENDO UM COMPOSTO CONTENDO ENXOFRE & A61K $31 / 145$ \\
\hline BR 1120180681555 & $\begin{array}{l}\text { 14/03/2017 COMPOSIÇÃO FARMACÊUTICA DE LIBERAÇÃO PROLONGADA QUE COMPREENDE CISTEAMINA } \\
\text { OU SAIS DA MESMA }\end{array}$ & $A_{A 61 K 9 / 50}$ \\
\hline BR 1020170018644 & $\begin{array}{l}\text { COMPOSTO QUÍMICO ÚTIL PARA REDUÇÃO DO VOLUME E/OU ALISAMENTO DE CABELOS, } \\
\text { 30/01/2017 PROCESSO DE OBTENÇÃO DO COMPOSTO, SOLUÇÃO ÚTIL PARA REDUÇÃO DO VOLUME E } \\
\text { ALISAMENTO DE CABELOS, PROCESSO DE OBTENÇÃO DE SOLUÇÃO, FORMULAÇÃO DE } \\
\text { PRODUTO REDUTOR DE VOLUME E/OU ALISANTE PARA OS CABELOS }\end{array}$ & A61K $8 / 44$ \\
\hline BR 1020160308178 & $\begin{array}{l}\text { 28/12/2016 IMUNOSSENSOR IMPEDIMÉTRICO PARA DETECÇÃO E QUANTIFICAÇÃO DE BACTÉRIAS } \\
\text { REDUTORAS DE SULFATO PELA PRESENÇA DA ENZIMA APS-REDUTASE }\end{array}$ & C12Q 1/04 \\
\hline BR 1020160158311 & $\begin{array}{l}\text { 07/07/2016 CATALISADORES HETEROGÊNEOS BASEADOS EM FILMES DE GRAFENO QUIMICAMENTE } \\
\text { MODIFICADO }\end{array}$ & $\operatorname{CO} 18$ 32/192 \\
\hline BR 1120170284480 & 01/07/2016 ANÁLOGOS DE CISTEAMINA RESISTENTES A ADO E USOS DOS MESMOS & A61K $31 / 145$ \\
\hline BR 1120170108720 & $\begin{array}{l}\text { 25/11/2015 CONJUGADOS DE CISTEAMINA DE ÁCIDO GRAXO E SEU USO COMO ATIVADORES DE } \\
\text { AUTOFAGIA }\end{array}$ & A61K $31 / 4425$ \\
\hline BR 1120170094487 & $\begin{array}{l}\text { MÉTODO PARA TRATAMENTO DA DOENÇA DE HUNTINGTON, MÉTODO PARA DESACELERAÇÃO } \\
\text { 05/11/2015 DA PROGRESSÃO DE ATROFIAS CEREBRAL E ESTRIATAL EM UM INDIVÍDUO ACOMETIDO DE } \\
\text { DOENÇA NEURODEGENERATIVA, E MÉTODO PARA TRATAMENTO DE DISTONIA EM UM } \\
\text { INDIVÍDUO ACOMETIDO DE UMA DOENÇA NEURODEGENERATIVA }\end{array}$ & A61P $25 / 28$ \\
\hline BR 1120170052725 & $\begin{array}{l}\text { 21/09/2015 USO DE CISTEAMINA NO TRATAMENTO DE INFECÇÕES CAUSADAS POR } \\
\text { LEVEDURAS/BOLORES }\end{array}$ & A61K $38 / 12$ \\
\hline
\end{tabular}

Fonte: Site do INPI (2019) 
Assim como nas bases de dados internacionais, o resultado da busca trouxe as diversas formas de se utilizar a Cisteamina, não somente na dermatologia, mas para tratamento capilar, infecções causadas por fungos e vírus, diabetes, cistinose, entre outros. Foram encontrados cerca de 24 registros de patentes, porém nenhuma para tratamento contra o melasma.

As principais empresas/instituições brasileiras que possuem registro de patentes com a substância Cisteamina são: Recordati Indústria Chimica e Farmacêutica s.p.a., com 04 registros, Horizon Orphan llc, com 04 registros, Ômega Bio-pharma International Limited, com 03 registros, Walcom Animal Science Limited, com 03 registros e Novabiotics Limited, com 02 registros.

\section{Levantamento Bibliográfico}

\subsection{Portal de Periódicos da Capes}

O Quadro 1 mostra os resultados da busca por publicações relacionadas à Cisteamina, realizada no Portal de Periódicos da Capes, no período de 1999 a 2019, ou seja, nos últimos 20 anos. Observa-se que as principais bases de dados que possuem o termo Cisteamina são: Scopus, Science Citation Index Expanded (Web of Science), MEDLINE/PubMed, Onefile e Science Direct Journals.

Quadro 1- Quantidade de publicações sobre a Cisteamina no Portal de Periódicos da Capes

\begin{tabular}{|c|c|}
\hline Palavra-chave & QuANTidadE DE PUBlicaçóEs \\
\hline Cysteamine & 10.420 \\
\hline Cisteamina & 36 \\
\hline "Cysteamine" AND "Melasma" & 13 \\
\hline "Cisteamina" AND "Melasma" & 0 \\
\hline
\end{tabular}

Fonte: Elaborada pelos autores deste artigo, (2019)

Analisando-se este resultado, constata-se um grande número apenas com o termo "cysteamine", pois esse ativo não é usado somente na dermatologia, mas também para tratamento da doença cistinose nefropática, esquizofrenia, úlcera duodenal, doença de Huntington, entre outras. A quantidade zero no resultado com os termos em português mostra que pesquisas científicas sobre a Cisteamina são todas em idiomas estrangeiros e que ainda são pesquisas recentes dentro da dermatologia. O resultado de quantidade de publicações em "Cysteamine" AND "Melasma", no caso são 13, revela que agora é que a dermatologia está pesquisando sobre utilizar a Cisteamina como antioxidante contra melasma. $\mathrm{O}$ artigo científico mais antigo relacionando o melasma com a Cisteamina é de 2013.

\subsection{Repositório Latino-Americano}

O Repositório Latino-Americano, da Universidade do Chile, agrupa as produções científicas de toda a América Latina e América Central. Esse portal permite que a pesquisa seja feita por instituições e é possível fazer a busca por publicações disponíveis, como teses, livros, artigos, entre outros. 
Quadro 2 - Quantidade de publicações sobre a Cisteamina no Repositório Latino-Americano

\begin{tabular}{|c|c|}
\hline Palavra-chave & Quantidade De PUBlicações \\
\hline Cysteamine & 108 \\
\hline Cisteamina & 56 \\
\hline "Cysteamine" AND "Melasma" & 0 \\
\hline "Cisteamina" AND "Melasma" & 0 \\
\hline
\end{tabular}

Fonte: Elaborado pelos autores deste artigo (2019)

Nota-se, no Quadro 2, que pesquisas relacionando o tratamento de melasma com a Cisteamina ainda não existem, o que mostra que ainda não existem estudos científicos no idioma em português. Já se utilizando as palavras-chave "Cysteamine" AND "Melasma" e "Cisteamina" AND "Melasma" verifica-se que não há nenhuma publicação de pesquisa. As produções científicas observadas com os termos "Cysteamine" e "Cisteamina" apresentaram resultado do uso da Cisteamina na medicina veterinária e também para tratamento da cistinose, no efeito antioxidante no desenvolvimento embrionário bovino e na prevenção de úlcera péptica.

\section{Discussão da Literatura}

Existem diversos estudos em relação ao uso da Cisteamina na medicina. Segundo a literatura pesquisada, essa substância também é utilizada para o tratamento de diversas doenças, como a esquizofrenia, doença renal como a cistinose, úlcera gástrica e hemorragias. Porém, este levantamento bibliográfico irá se restringir ao uso da Cisteamina no combate ao melasma resistente. A eficácia do medicamento Cisteamina tópico no tratamento do melasma foi pesquisada por diversos cientistas nos últimos anos.

O melasma é uma disfunção adquirida e crônica da melanogênese, resultando em hiperpigmentação da pele caracterizada por máculas e manchas hipercrômicas marrons com bordas irregulares que ocorrem predominantemente no rosto, mas pode se manisfestar na região do colo e braços (CESTARI; PERUZZO; GIONGO, 2017).

Segundo Saraiva et al. (2018), o melasma é hiperpigmentação crônica adquirida, acometendo áreas fotoexpostas da pele. Incide em todas as raças e em ambos os sexos, predominando em mulheres em idade fértil e de fototipos mais elevados. Sua prevalência é de $1 \%$ a $50 \%$ da população, variação decorrente da etnia e do índice de radiação ultravioleta.

De acordo com Hsu, Mahdi e Pourahmadi (2013), a Cisteamina é um ativo conhecido como um potente despigmentador há mais de cinco décadas. Apesar de seu forte efeito despigmentador, ela se mostrou mais efetiva do que a hidroquinona. Recentemente, uma nova tecnologia foi disponibilizada, permitindo a redução considerável do odor da Cisteamina. Então, foi desenvolvido o creme de Cisteamina que mostrou um forte efeito clareador na pele, confirmando considerável eficácia no tratamento do melasma.

O melasma é um distúrbio hiperpigmentar de difícil tratamento. Embora a Cisteamina seja um agente despigmentante potente conhecido, a sua eficácia no tratamento do melasma não foi testada. O tratamento com creme de Cisteamina encontrou o resultado primário de diminuir o conteúdo de melanina das lesões após 2 e 4 meses de aplicação, demonstrando sua eficácia 
no melasma epidérmico. A discussão da literatura indica que o presente estudo pode ser o primeiro ensaio clínico controlado que examinou a eficácia do creme de Cisteamina no tratamento de um distúrbio hiperpigmentar em seres humanos (MANSOURI; FARSHI; HASHEMI, 2014).

A Cisteamina é um antioxidante biológico produzido durante o ciclo de metabolismo da coenzima A e está naturalmente presente em todas as células de mamíferos. A eficácia da Cisteamina tópica no tratamento do melasma foi recentemente demonstrada em dois ensaios clínicos duplo-cegos, randomizados e controlados com placebo. Aqui, nós relatamos um paciente de 44 anos com melasma resistente à fórmula de Kligman (creme Pigmanorm), que foi tratado com sucesso com Cisteamina tópica como um novo agente despigmentante. Medidas colorimétricas da pele, determinação do escore MASI e fotografias padrão após 2 e 4 meses de aplicação diária de creme de Cisteamina mostraram uma melhora acentuada das lesões hiperpigmentadas. A telangiectasia e a hipopigmentação perilesional melhoraram rapidamente após a descontinuação da fórmula de Kligman e o início do tratamento com Cisteamina tópica. Após 4 meses, os resultados terapêuticos foram mantidos através de um regime de aplicação quinzenal de creme de Cisteamina. O uso de creme de Cisteamina foi bem tolerado e não induziu efeitos colaterais durante o acompanhamento de 3 anos do paciente. A Cisteamina é uma molécula natural com excelente perfil de segurança e efeitos antimutagênicos, antimelanoma e anticarcinogênicos conhecidos. Considerando-se a alta eficácia do creme de Cisteamina, é possível que ele substitua agentes despigmentantes mutagênicos e carcinogênicos, como a hidroquinona, em um futuro próximo (KASRAEE; MANSOURI; FARSHI, 2018).

Melasma é um distúrbio de hiperpigmentação comum, terapeuticamente desafiador e universalmente recorrente, que é mais frequentemente observado em mulheres e indivíduos com tipos de pele de Fitzpatrick III a VI. A patogênese do melasma é complexa e protéica. Fatores contribuintes que são frequentemente implicados na etiopatogenia dessa condição incluem uma predisposição genética, exposição intensa à radiação ultravioleta e influências hormonais. Intervenções terapêuticas para o melasma incluem uma abordagem multimodalidade incorporando agentes de fotoproteção, clareadores de pele tópicos e orais e procedimentos de resurfacing. Tratamentos novos e eficazes estão expandindo o arsenal terapêutico, analisando novos e emergentes tratamentos orais e tópicos para o melasma (GRIMES; IJAZ; NASHAWATI, 2019).

No estudo de Mansouri et al. (2018), o melasma é um distúrbio hiperpigmentar de difícil tratamento. Poucos estudos foram realizados sobre a eficácia da Cisteamina no tratamento do melasma. E, o creme de Cisteamina mostrou eficácia significativa na diminuição do conteúdo de melanina das lesões.

\section{Considerações Finais}

Neste artigo, cujo objetivo era realizar uma análise prospectiva das patentes de Cisteamina depositadas no Brasil e no exterior, levando-se em consideração os principais depositantes, em uma evolução temporal de 20 anos, optou-se por utilizar o método de prospecção por meio de patentes, por ser uma potente ferramenta de monitoramento, sistemas de inteligência, ser uma fonte confiável de informação, ter padronização, além de dar apoio à tomada de decisão. Esta metodologia permite identificar tecnologias relevantes, parceiros, nichos de mercado para atuação e inovações incrementais e movimentos de concorrência. 
O levantamento bibliográfico em artigos científicos e a discussão da literatura abordados neste trabalho mostram que há baixa produção de artigos publicados no Brasil, sendo autores estrangeiros os que mais publicam produção científica sobre o tema pesquisado.

Melasma é difícil de tratar o distúrbio hiperpigmentar, e muitos pacientes mostram resistência aos produtos despigmentantes atualmente disponíveis. A hidroquinona tem sido usada como tratamento padrão-ouro para o melasma desde 1950. Já a Cisteamina é um antioxidante intracelular natural que é produzido em quase todas as células de mamíferos. As células do corpo produzem cisteamina (KASRAEE; MANSOURI; FARSHI, 2019).

A partir dos resultados, observa-se que o uso da Cisteamina como mais um agente de tratamento ao melasma é recente. Este estudo não teve o intuito de esgotar as discussões sobre as temáticas apresentadas, mas sim de fomentar o debate. Desse modo, recomenda-se como estudos futuros a descrição de outras técnicas dermatológicas e cosméticas para auxiliar no tratamento desse distúrbio pigmentar que acomete principalmente mulheres onde a incidência solar é elevada, agravando assim a eliminação definitiva do melasma.

\section{Referências}

CESTARI, T.; PERUZZO, J.; GIONGO, N. Definition, incidence and etiology of melasma in brown skin. In: Melasma and vitiligo in brown skin. Springer, New Delhi, 2017. p. 13-19.

CISTEAMINA HCL: ativo clareador para controle do melasma. Primacêutica, 2018. Disponível em: http://www.primaceutica.com.br/wp-content/uploads/2019/04/Cisteamina-HCL.pdf. Acesso em: 02 jul. 2019.

DREHMER, Raquel. Cisteamina e microagulhamento: o que há de novo no tratamento para melasma. Revista MdeMulher, 2019. Disponível em: https://mdemulher.abril.com.br/saude/ cisteamina-e-microagulhamento-o-que-ha-de-novo-no-tratamento-para-melasma/. Acesso em: 14 de jul. 2019.

GHEISARI, Mehdi; DADKHAHFAR, Sahar; OLAMAEI, Elham. The efficacy and safety of topical $5 \%$ methimazole vs $4 \%$ hydroquinone in the treatment of melasma: A randomized controlled Trial.

Journal of Cosmetic Dermatology, 2019. Disponível em: https://onlinelibrary-wiley.ez7.periodicos. capes.gov.br/doi/full/10.1111/jocd.12987. Acesso em: 19 jul. 2019.

GRIMES, P. E.; IJAZ, S.; NASHAWATI, R. New oral and topical approaches for the treatment of melasma. International Journal of Women's Dermatology, v. 5, p. 30-36, 2019. Disponível em: https://www-sciencedirect.ez7.periodicos.capes.gov.br/journal/international-journal-of-womensdermatology/vol/5/issue/1. Acesso em: 14 jul. 2019.

HSU, Christophe; MAHDI, Hussain Ali; POURAHMADI, Mohammad. Cysteamine cream as a new skin depigmenting product. Journal of the American Academic of Dermatology, v. 68, n. 4, 2013. Disponível em: https://www.jaad.org/article/S0190-9622(12)02060-9/abstract. Acesso em: 14 jul. 2019.

HUERTH, Kimberly; SHAHZEB, Hassan; CALLENDER, Valerie. Therapeutic insights in melasma and hyperpigmentation management. Journal of Drugs in Dermatology, v. 18, n. 8, p. 718-729, ago. 2019. Disponivel em: https://jddonline.com/articles/dermatology/S1545961619P0718X/1/. Acesso em: 2 set. 2019. 
INSTITUTO NACIONAL DA PROPRIEDADE INDUSTRIAL - INPI. Disponível em: http://www.inpi. gov.br/. Acesso em: 16 jul. 2019.

KASRAEE, Behrooz; MANSOURI, Parvin; FARSHI, Susan. Significant therapeutic response to cysteamine cream in a melasma patient resistant to Kligman's formula. Journal of Cosmetic Dermatology, v. 18, n. 1, p. 293(3), 2019. Disponível em: https://onlinelibrary-wiley.ez7.periodicos. capes.gov.br/doi/full/10.1111/jocd.12837. Acesso em: 15 jul. 2019.

MAGALHÃES, Cláudia. O que há de novo em melasma segundo o Meeting AAD 2018? 2018. Disponível em: https://noticias.ne10.uol.com.br/coluna/questao-de-pele/noticia/2018/02/28/o-queha-de-novo-em-melasma-segundo-o-meeting-aad-2018-723241.php. Acesso em: 12 jul. 2019.

MANSOURI, P.; FARSHI, S.; HASHEMI, Z. Evaluation of the efficacy of cysteamine $5 \%$ cream in the treatment of epidermal melasma: a randomized double $\square$ blind placebo $\square$ controlled Trial. British Journal of Dermatology, v. 173, n. 1, p. 209-2017, 2015. Disponível em: https://onlinelibrarywiley.ez7.periodicos.capes.gov.br/doi/full/10.1111/bjd.13424. Acesso em: 15 jul. 2019.

MANSOURI, P.; FARSHI, S.; HASHEMI, Z. Efficacy of cysteamine cream in the treatment of epidermal melasma, evaluating by Dermacatch as a new measurement method: a randomized double blind placebo controlled study. Journal of Dermatological Treatment, v. 29, n. 2, p.182190, 2018. Disponível em: https://www.unboundmedicine.com/medline/citation/28678558/Efficacy_ of_cysteamine_cream_in_the_treatment_of_epidermal_melasma_evaluating_by_Dermacatch_as_a_ new_measurement_method:_a_randomized_double_blind_placebo_controlled_study_. Acesso em: 15 jul. 2019.

MEDICAMENTO: cisteamina é novidade para melasma. Folha Vitória, maio 2019. Disponível em: https:/www.folhavitoria.com.br/saude/noticia/05/2019/medicamento-cisteamina-e-novidade-paramelasma. Acesso em: 10 jul. 2019.

MUNOZ, Begona Escutia; ESTEBANEZ, Esther Quecedo; ESTRADA, Rafael Botella. Tratamiento del melasma. Piel, v. 25, n. 07, p. 405-410, 2010. Disponível em: https://www-sciencedirect.ez7. periodicos.capes.gov.br/search/advanced?docld=10.1016/j.piel.2010.01.005. Acesso em: 19 jul. 2019.

- RED DE REPOSITÓRIOS LATINO AMERICANOS. Disponível em: https:// repositorioslatinoamericanos.uchile.cl/. Acesso em: 11 jul. 2019.

SARAIVA, Luciana; NASCIMENTO, Mayara; FILIPPO, Alexandre. Tratamento de melasma facial com associação do microagulhamento robótico e drug delivery de ácido tranexâmico. Surgical and Cosmetic Dermatology, v. 10, n. 4, 2018. Disponível em: http://www.surgicalcosmetic.org.br/ detalhe-artigo/685/Tratamento-de-melasma-facial-com-associacao-do-microagulhamento-robotico-edrug-delivery-de-acido-tranexamico. Acesso em: $1^{\circ}$ jul. 2019.

SITONIO, Renata. Congressos internacionais de dermatologia apresentam cisteamina como arma poderosa para tratar melasma. Newslab, ano 25, ago./set. 2018. Disponível em: https://https:// newslab.com.br/congressos-internacionais-de-dermatologia-apresentam-cisteamina-como-armapoderosa-para-tratar-melasmas/. Acesso em: 5 jul. 2019.

TACKLING MELASMA. 2017. Disponível em: skingab.com/tackling-melasma/. Acesso em: $1^{\circ}$ jul. 2019.

VARELLA, Maria Helena. Melasma: doenças e sintomas. Disponível em: https://drauziovarella.uol. com.br/doencas-e-sintomas/melasma/. Acesso em: $1^{\circ}$ set. 2019. 


\section{Sobre os Autores}

\section{Marília Dione Salvador Shibayama}

E-mail:mardioli3@gmail.com

Especialização em Gestão da Informação em Bibliotecas Digitais pela UFPA.

Endereço profissional: Rod. Juscelino Kubitschek, km 02, Jardim Marco Zero, Macapá, AP. CEP: 68903-419.

\section{Geraldo Neves de Albuquerque Maranhão}

E-mail: geraldo.maranhao@gmail.com

Doutorado em Engenharia Elétrica pela UFPA.

Endereço profissional: Rod. Juscelino Kubitschek, km 02, Jardim Marco Zero, Macapá, AP. CEP: 68903-419.

\section{Werbeston Douglas de Oliveira}

E-mail:wdoliveira@unifap.com

Doutorado em Engenharia Elétrica pela UFPA.

Endereço profissional: Rod. Juscelino Kubitschek, km 02, Jardim Marco Zero, Macapá, AP. CEP: 68903-419. 